\title{
Long VA tachycardia after slow pathway modification for slow-fast atrioventricular nodal reentrant tachycardia : Insights about the mechanism from intriguing electrogram and polarity reversal.
}

\author{
Debabrata Bera ${ }^{1}$, Ayan Kar ${ }^{1}$, Radhey Shyam Joshi ${ }^{1}$, and Biswajit Majumder ${ }^{2}$ \\ ${ }^{1}$ RTIICS \\ ${ }^{2} \mathrm{RG}$ Kar Medical College
}

April 28, 2020

\section{Title page}

Title : Long VA tachycardia after slow pathway modification for slow-fast atrioventricular nodal reentrant tachycardia : Insights about the mechanism from intriguing electrogram and polarity reversal.

Short Title: Mechanism of long VA tachycardia after slow pathway modification.

Authors:

Debabrata Bera, DM, Division of Electrophysiology, Dept of Cardiology, RTIICS, Kolkata. India (Place of the study).

Ayan Kar, DNB, Dept of Cardiology, RTIICS, Kolkata, India.

Radhey Shyam Joshi, DM, HOD, Dept of Cardiology, RTIICS, Kolkata, India.

Biswajit Majumder, DM, Dept of Cardiology, RG Kar Medical college and Hospital, Kolkata, India.

\section{Corresponding author:}

Debabrata Bera. Rabindranath Tagore International Institute of Cardiac Sciences (RTIICS), 124 Mukundapur, Kolkata. West Bengal, India. PIN- 700099.

Ph: +91-8013894181, Fax: +91-33 2426 4204.Email- debu2000pgi@gmail.com

Key words: atypical AVNRT; fast slow AVNRT; long VA tachycardia after slow pathway modification; polarity reversal.

Funding: None

Conflict of interest: None

Acknowledgements: None.

Word count -1145 .

Case

A 45-year-old lady presented with recurrent narrow QRS tachycardia. Echocardiography was normal. During EP study dual AV nodal physiology was demonstrated: with PR interval exceeding RR interval during decremental pacing, AH jump and AV nodal echo (slow-fast). It was followed by easily inducible typical slow-fast atrioventricular nodal reentrant tachycardia (AVNRT, tachy1, septal VA=0 ms) [Post ventricular 
entrainment- VAHV response, Stim A - VA=125 ms, corrected post-pacing interval-tachycardia cycle length $=116 \mathrm{~ms}$ ] (Fig 1A,1B). After slow pathway (SP) modification in lower triangle of Koch, (although only very few junctional beats were generated in 4 radiofrequency (RF) lesions, total $150 \mathrm{sec}$ of RF energy), during decremental pacing PR interval didn't exceed RR interval, no AH jump, no typical echo; but consistently had one and half long VA echo(Fig2A). After isoprenaline, sustained tachycardia with similar long VA (septal $V A=144 \mathrm{~ms}$ ) was induced (Fig 2A). Attempted ventricular overdrive pacing (VOP) to entrain the tachycardia repeatedly terminated tachycardia (Fig 2C) without entraining tachycardia even after 7-8 beats.

\section{Q > What is the second tachycardia (tachy2) and how to proceed?}

1. Fast-slow AVNRT

2. Slow-slow AVNRT

3. Slow fast AVNRT

4. Orthodromic AVRT

5. Atrial tachycardia

6. Junctional Tachycardia

\section{Commentary:}

With the given clinical background atypical AVNRT was the most likely possibility. But maneuvers were needed to prove or exclude other diagnoses. Para his pacing (PHP) in sinus rhythm was suggestive nodal route for VA conduction. His-refractory PVC didn't perturb the tachycardia circuit. Junctional tachycardia (JT) was ruled out as single late atrial premature depolarization (APD) could reproducibly terminate tachy2. VOP repeatedly terminated tachycardia. VOP could not entrain the SVT/atrium (even for a single beat), even after 7-8 beats before termination, hence, ruling out AVRT. VOP repeatedly terminated the tachy2 without conduction to A (third beat in Fig2C), thus, ruling out AT. With AVNRT as the only diagnosis of exclusion, further slow pathway modification was performed. Tachy2 became non-inducible afterwards. No jump or AV echo was produced at baseline. With isoprenaline, single typical AV nodal echo beat noted (with PR of $360 \mathrm{~ms}$ ).

Q: After initial SP modification, why the initial tachy1 became non-inducible, and tachy2 got induced?

Ans. The exact mechanism is difficult to predict, like various other mysteries of AVNRT [1]. We hypothesize, with SP modification the ERP of SP and/or FP had probably changed such that fast-slow/ slow-slow AVNRT was easily induced without $\mathrm{AH}$ jump.

Is it fast-slow AVNRT?

Yes. Most likely as the retrograde A was earliest near CS os. A possible reason is increase in effective refractory period (ERP) of SP after ablation. This is known after SP modification [2]. Hence, with APD, ERP of SP was reached earlier than FP (unlike pre-ablation, when normally ERP of FP is reached before SP, producing unidirectional block in FP leading to jump to SP and typical echo; finally perpetuating slow-fast AVNRT). We hypothesize, now, with the unidirectional block happening earlier in SP, it was still conducted via $\mathrm{FP}$ (ERP of $\mathrm{FP}$ is also known to shorten with SP modification) [2]. By that time SP recovered and was available to conduct retrogradely leading to fast-slow AVNRT.

Can it be slow-slow AVNRT?

There was no AH jump and only modest $\mathrm{PR}$ interval $(\mathrm{PR}=190 \mathrm{~ms})$. So antegrade limb is very unlikely to be SP.

Can it be still be slow-fast AVNRT?

No, very unlikely. The retrograde limb is definitely SP as the earliest 'A' in tachy2 was near CS ostium [3].

Q > Any further insight from the attempted VOP about the mechanism whether it's slow-fast, fast-slow, or slow-slow? 
Ans. Let's have a look at the 4 atrial activation sequences in CS catheter during:

a. Initial AVNRT (tachy1)

b. First VOP1

c. Second tachycardia and

d. Attempted VOP (VOP2).

There is very little doubt that during tachy1 the antegrade limb is SP and the retrograde limb is FP. The CS activation is sequentially 'CS78CS12'. During VOP entrainment of tachy1 could be achieved with VAHV response. In the entrained beats also, the CS activation was similar i.e. 'CS78CS12'; suggesting same VA route $(\mathrm{FP})$ during entrainment which is the norm in typical AVNRT.

Now during the longer VA tachycardia (tachy2), the possibility of fast slow AVNRT is more likely as explained above (no critical AH jump noted, at or before induction). But more insights resurfaced when the atrial activation was looked into carefully. The CS activation was different there than tachy1. The timing of 'A' was near simultaneous in CS 78 and CS56 suggestive of a retrograde breakthrough at an equidistant point from either of the bipole (Fig 2B). Moreover, there was polarity reversal (between CS 78 and CS 56) of the initial segment of the triphasic/quadriphasic 'A' electrogram (EGM)(Fig 2B). In addition, the His proximal pole also had similar timing of 'A' signal compared to CS78/CS56. These unequivocally proved the retrograde circuit was different from FP (tachy1 and VOP1).

As described above, VOP2 repeatedly terminated the tachy2. During VOP the tachy2 had terminated without conduction to atrium ( $3^{\text {rd }}$ beat in Fig $2 \mathrm{C}$ ). The tachy 2 continued for several beats after starting VOP (till $2^{\text {nd }}$ beat of RV pacing in Fig $2 \mathrm{C}$ ). In the $3^{\text {rd }}$ beat captured ventricle has terminated the tachy 2 by entering the excitable gap. From $3^{\text {rd }}$ beat onwards it is merely a VA conduction happening after tachycardia termination, which is clear from AA interval measurements (Fig 2C). In addition, if we look at the EGM of CS, till the tachycardia was continuing, the 'A' EGM in CS 56 and CS78 was simultaneous (CS78=CS56 , first 2 beats in Fig2C). But as soon as tachy2 terminated (and there was VA conduction during continued RV pacing), the CS activation pattern was different, but was identical to tachy1and VOP1 (Fig 1B), hence now via FP (CS78CS56). This again proves that the retrograde limb of the tachy2 was anything other than FP and fast-slow AVNRT becomes the only possible mechanism. In fact, the EGM in CS78 itself changed significantly between tachy 2 and VA conduction. The initial vector of +/- (marked as * in Fig $2 \mathrm{C}$ ) became negative of higher amplitude (** in Fig $2 \mathrm{C}$ ). This is also another kind of polarity reversal, and was due to change in the route of VA conduction.

In essence, this interesting case highlights importance of stepwise application of maneuvers to confirm or exclude SVT mechanisms. Minute EGM signal change and polarity reversal can also provide useful insights in determining exact AVNRT mechanism.

\section{Figure legend}

Fig 1: A: Induction of typical AVNRT (tachy1, with APD). B: successful entrainment with VOP and long cPPI-TCL and StimA- VA.

Fig 2A: After initial slow pathway modification for tachy1, this one and half beat of long VA (septal VA 164 ms) echo was noted reproducibly at baseline.

2B: With isoprenaline, sustained tachy2 was induced with long VA ( VA= $144 \mathrm{~ms})$ similar to the abovementioned echo beat.

2C: One of the attempted VOP. During initial few beats the tachy2 (AA=340 ms) continued at its own rate $\left(\mathrm{TCL}=340 \mathrm{~ms}\right.$ ) as seen in first 2 beats in Fig $2 \mathrm{C}$. In the $8^{\text {th }}$ beat of the drive train $\left(3^{\text {rd }}\right.$ beat in fig 2C) of VOP @320 ms, the AA interval suddenly prolongs by 23ms as compare to TCL (340 ms), suggesting termination of the tachy2. After termination AA interval was same as RV pacing CL @320 ms. Noted the 
change in CS78-CS56 A-EGM relation during tachycardia (first 2 beats) and VA conduction (last 2 beats). The polarity reversal was noted in A - EGM of CS 78 .

(TCL- tachycardia cycle length, CL- cycle length)

CS- coronary sinus catheter, CS78- proximal CS, CS 12- distal CS, His D- His catheter distal pole, His PHis catheter proximal pole, Map - Mapping catheter in right ventricle, STIM- Stimulation.)

\section{Reference:}

1. Lockwood D, Nakagawa H, Jackman WM. Electrophysiologic characteristics of atrioventricular nodal reentrant tachycardia: implications for reentrant circuits. In: , Zipes DP, Jalife J, eds. Cardiac Electrophysiology: From Cell to Bedside. 5th ed. Philadelphia, PA: W.B. Saunders; 2009:615-656.

2. Kim JY, Kim S-H, Kim T-S, Kim J-H, Jang SW, Oh YS, et al. Changes in Atrioventricular Node Physiology Following Slow Pathway Modification in Patients with AV Nodal Re-entrant Tachycardia: The Hypothetical Suggestion of Mechanism of Noninducibility of AVNRT. Int J Arrhythmia. 2016 Mar 29;17(1):6-13.

3. Nawata H, Yamamoto N, Hirao K, Miyasaka N, Kawara T, Hiejima K, Harada T, Suzuki F. Heterogeneity of anterograde fast-pathway and retrograde slow-pathway conduction patterns in patients with the fast-slow form of atrioventricular nodal reentrant tachycardia: electrophysiologic and electrocardiographic considerations.J Am Coll Cardiol. 1998; 32 :1731-1740.

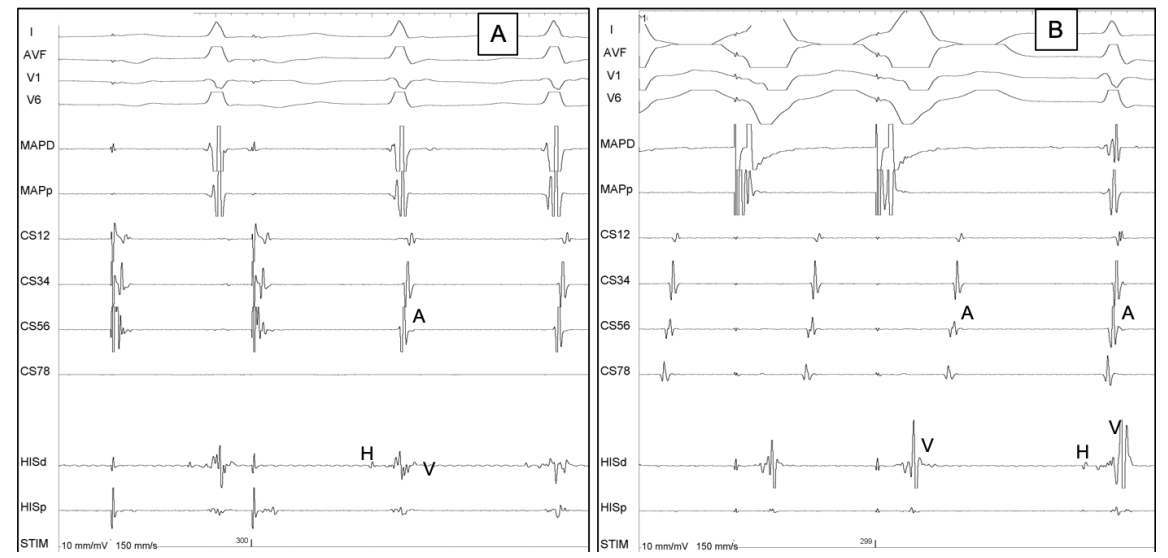




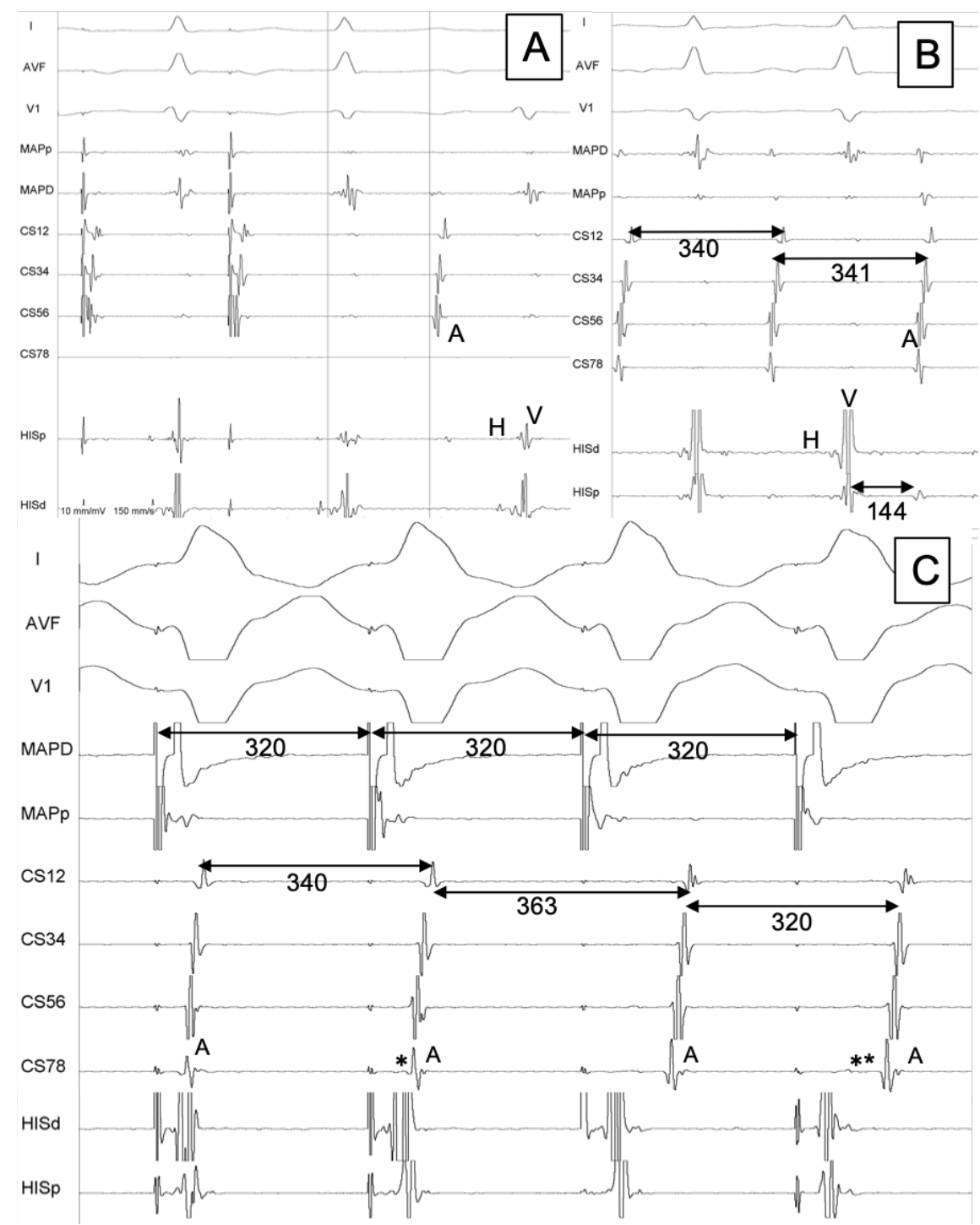

